\title{
Power law relaxation in a complex system: Omori law after a financial market crash
}

\author{
F. Lillo* and R. N. Mantegna*,† \\ * Istituto Nazionale per la Fisica della Materia, Unità di Palermo, Viale delle Scienze, I-90128, Palermo, Italia \\ † Dipartimento di Fisica e Tecnologie Relative, Università di Palermo, Viale delle Scienze, I-90128 Palermo, Italia.
}

\begin{abstract}
We study the relaxation dynamics of a financial market just after the occurrence of a crash by investigating the number of times the absolute value of an index return is exceeding a given threshold value. We show that the empirical observation of a power law evolution of the number of events exceeding the selected threshold (a behavior known as the Omori law in geophysics) is consistent with the simultaneous occurrence of (i) a return probability density function characterized by a power law asymptotic behavior and (ii) a power law relaxation decay of its typical scale. Our empirical observation cannot be explained within the framework of simple and widespread stochastic volatility models.
\end{abstract}

PACS numbers: 89.65.-s,89.75.-k

Several complex systems are statistically characterized by power-law distributions. Examples are earthquakes, financial markets, landslides, forest fires and scale free networks. Power law distributions imply that rare events are occurring with a finite non-negligible probability in complex systems. It is therefore meaningful to ask the following scientific question: how is the dynamics of a complex system affected when the system undergoes to an extreme event? An answer to this question concerning earthquakes was provided by Omori more than a century ago [1]. The Omori law describes the non stationary period observed after a big earthquake. In his study, the number of aftershocks per unit of time is described by a power law and a time scale for the relaxation process of the complex system to its typical state does not exist. Non exponential relaxation to a typical state has also been observed in several physical and social systems. For example, power law relaxation has been theoretically predicted and experimentally observed in spin glasses [2], condensed matter systems [3], microfracturing phenomena [4], physical systems described by a fractional FokkerPlanck equation [5], in the kinetics of reversible bimolecular reactions [6], in two-dimensional arrays of magnetic dots interacting by long-range dipole-dipole interactions [7], in the Internet dynamical response [8] and in the Internet traffic [9].

In the present study we investigate the dynamics of a model complex system when it is moved far away from its typical state by the occurrence of an extreme event. This is done by investigating the statistical properties of time series of financial indices in the time period immediately after a financial crash. These market phases are indeed strongly non-stationary and we show that a time power law relaxation is detected when the financial market is moved far away from its typical behavior.

Financial time series of stock or index returns are modeled in terms of random processes 10, 11]. Empirical investigations show that the time series of stock or index return is not strictly sense stationary. In fact the volatility of the financial asset, i.e. the standard deviation of asset returns describing the typical scale of the process, is itself a stochastic process fluctuating in time [12, 13].
The non-stationary evolution of asset returns can sometimes show relaxation time patterns. Specifically, decaying patterns of volatility are observed in time periods immediately after a financial crash. An illustrative example of such non-stationary time pattern is given in Fig. 1 where we plot the one-minute logarithm changes of the index $r(t)$ (a quantity essentially equivalent to return) for the Standard and Poor's 500 (S\&P500) index during 100 trading days after the Black Monday (19 October 1987). The pattern observed in Fig. 1 is not invariant under time-reversal. Other examples of statistical properties of market which are not time-reversal have been observed in the investigation of cross-sectional quantities computed for a set of stocks before and after financial crashes [14].

A direct characterization of the time evolution of the scale of the random process of return is extremely difficult in financial markets and in several other complex systems due to the fact that the random variable is highly fluctuating and that system is unavoidably monitored by just recording a single random realization. We make use of a different and statistically more robust method. Specifically, we quantitatively characterize the time series of index returns in the non-stationary time period by investigating the number of times $|r(t)|$ is exceeding a given threshold value. This investigation is analogous to the investigation of the number $n(t)$ of aftershock earthquakes measured at time $t$ after the main earthquake. The Omori law $n(t) \propto t^{-p}$ says that the number of aftershock earthquakes per unit time measured at time $t$ after the main earthquake decays as a power law. In order to avoid divergence at $t=0$ Omori law is often rewritten as

$$
n(t)=K(t+\tau)^{-p}
$$

where $K$ and $\tau$ are two positive constants. An equivalent formulation of the Omori law more suitable for comparison with real data can be obtained by integrating equation (1) between 0 and $t$. In this way the cumulative number of aftershocks observed until time $t$ after the main earthquake is

$$
N(t)=K\left[(t+\tau)^{1-p}-\tau^{1-p}\right] /(1-p)
$$


when $p \neq 1$ and $N(t)=K \ln (t / \tau+1)$ for $p=1$. The value of the exponent $p$ for earthquakes ranges between 0.9 and 1.5. Because $N(t)$ is related to $n(t)$ by a summation, the fluctuation in $N(t)$ is substantially reduced compared with the fluctuation in $n(t)$. Hence customary measurement of $N(t)$ leads to a more reliable characterization of the aftershock period than measurement of $n(t)$.

We first investigate the index returns during the time period after the Black Monday crash (19 October 1987) occurred at New York Stock Exchange (NYSE). This crash was one of the worst crashes occurred in the entire history of NYSE. The S\&P500 went down $20.4 \%$ that day. In our investigation, we select a 60 day after crash time period ranging from 20 October 1987 to 14 January 1988. This time period is chosen to maximize the time period investigated by simultaneously ensuring that the relaxation process is still going on. The selected value is not a critical one and time windows of 50 or 70 trading days provide similar results. For the selected time period, we investigate the one-minute return time series of the S\&P500 Index. The first estimate concerns the unconditional one-minute volatility which is equal to $\sigma=4.91 \times 10^{-4}$. In Fig. 2 we show the cumulative number of events $N(t)$ detected by considering all the occurrences observed when the the absolute value of index return exceeds a threshold value $\ell$ chosen as $4 \sigma, 5 \sigma, 6 \sigma$ and $7 \sigma$. For all the selected threshold values we observe a nonlinear behavior. Nonlinear fits performed with the functional form of equation (2) well describes the empirical data for the entire time period. This paradigmatic behavior is not specific of the Black Monday crash of the S\&P 500 index. In fact, we observe similar results also for a stock price index weighted by market capitalization for the time periods occurring after the 27 October 1997 and the 31 August 1998 stock market crashes. This index has been computed selecting the 30 most capitalized stocks traded in the NYSE and by using the highfrequency data of the Trade and Quote database issued by the NYSE. In Fig. 3 we show $N(t)$ for $\ell=4 \sigma$ where $\sigma$ is again the unconditional one-minute volatility in the considered periods. We estimate $\sigma=4.54 \times 10^{-4}$ during the period from 28 October 1997 to 23 January 1998 and $\sigma=6.09 \times 10^{-4}$ during the period from 1 September 1998 to 24 November 1998. In the left part of Table I, we summarize the values of the $p$ exponents obtained by best fitting with equation (2) the cumulative number of events exceeding the selected threshold values for the considered market crashes. The value of the exponent $p$ varies in the interval between 0.70 and 0.99 . The estimate of the exponent $p$ is slightly increasing when the threshold value $\ell$ is increasing. Below we will comment the relation between this observation and the properties of the index return probability density function (pdf). The detected nonlinear behavior of $N(t)$ is specific to aftercrash market period. In fact an approximately linear behavior of $N(t)$ is observed when a market period of roughly constant volatility such as, for example, the 1984 year is investigated. This is due to the fact that when the process is stationary the frequency of aftershock $n(t)$ is on average constant in time and therefore the cumulative number $N(t)$ increases linearly in time. In terms of equation (2) this implies that the exponent $p$ is equal to zero. For independent identically distributed random time series it is possible to characterize $n(t)$ in terms of an homogeneous Poisson process 15]. The results summarized in the left part of Table I imply that the time period immediately after a big market crash has statistical properties which are different from constant volatility periods. In particular, index return cannot be modeled in terms of independent identically distributed random process after a big market crash.

The empirical evidence of the power-law decrease of the frequency of aftershocks is consistent with a powerlaw decay of volatility after a major crash. In order to prove this claim, we describe the empirical behavior of $N(t)$ by assuming that during the time period after a big crash the stochastic variable $r(t)$ is the product of a time dependent scale $\gamma(t)$ times a stationary stochastic process $r_{s}(t)$. For the sake of simplicity, we also assume that the pdf of $r(t)$ is approximately symmetrical. Under these assumptions, the frequency of events of $|r(t)|$ larger than $\ell$ observed at time $t$ is

$$
n(t) \propto 2 \int_{\ell}^{+\infty} f(r, t) d r
$$

where $f(r, t)$ is the pdf of $r(t)$ at time $t$. One can rewrite equation (3) in terms of the cumulative distribution function $F_{s}\left(r_{s}\right)$ of the random variable $r_{s}(t)$ as

$$
n(t) \propto 1-F_{s}(\ell / \gamma(t)) .
$$

In this description, the specific form of the time evolution of $n(t)$ is, for large values of the threshold $\ell$, controlled by the properties of (i) the time evolution of the scale $\gamma(t)$ and (ii) the asymptotic behavior of the pdf for large values of $\left|r_{s}(t)\right|$.

By assuming that the stationary return pdf behaves asymptotically as a power law

$$
f_{s}\left(r_{s}\right) \sim \frac{1}{r_{s}^{\alpha+1}}
$$

the frequency of events $n(t)$ becomes for large values of $\ell$

$$
n(t) \sim(\gamma(t) / \ell)^{\alpha} .
$$

By hypothesizing that $\gamma(t) \sim \exp (-k t)$, the frequency of events above threshold is expected exponentially decreasing $n(t) \sim \exp (-\alpha k t)$. Conversely, when the scale of the stochastic process decays as a power law $\gamma(t) \sim$ $t^{-\beta}$, the frequency of events above threshold is power law decaying as $n(t) \sim 1 / t^{p}$. It is worth noting that the exponent $p$ is given by

$$
p=\alpha \beta
$$


TABLE I: Exponents obtained from the empirical analyses of 60 day market periods occurring after the 19 October 1987,27 October 1997 and 31 August 1998 market crashes.

\begin{tabular}{|c|c|c|c|c|c|c|c|}
\hline & \multicolumn{4}{|c|}{$p$} & \multirow[t]{2}{*}{$\alpha$} & \multirow[t]{2}{*}{$\beta$} & \multirow[t]{2}{*}{$\alpha \beta$} \\
\hline & $4 \sigma$ & $5 \sigma$ & $6 \sigma$ & $7 \sigma$ & & & \\
\hline 1987 & 0.85 & 0.90 & 0.99 & 0.99 & $3.18 \pm 0.34$ & $0.32 \pm 0.02$ & $1.02 \pm 0.13$ \\
\hline 1997 & 0.70 & 0.73 & 0.73 & 0.76 & $3.67 \pm 0.40$ & $0.22 \pm 0.04$ & $0.81 \pm 0.17$ \\
\hline 1998 & 0.99 & 0.99 & 0.99 & 0.99 & $3.49 \pm 0.37$ & $0.32 \pm 0.05$ & $1.12 \pm 0.21$ \\
\hline
\end{tabular}

The previous relation links the exponent $p$ governing the number of events exceeding a given threshold to the $\alpha$ exponent of the power law return cumulative distribution and to the $\beta$ exponent of the power law decaying scale. It is worth noting that a power law behavior of the return pdf is observed only for large absolute values of returns. Hence, the relation between exponents (eq. $(7))$ is valid only for large values of the threshold $\ell$ used to determine the exponent $p$. Our theoretical considerations show that a number of events above threshold decaying as a power law, i.e. the analogous of the Omori law, is consistent with the simultaneous occurrence of: (i) a return pdf characterized by a power law asymptotic behavior and (ii) a non-stationary time evolution of the return pdf whose scale is decaying in time as a power law. These hypotheses are consistent with recent empirical results. In fact, a return pdf characterized by a power law asymptotic behavior has been observed in the price dynamics of several stocks [16, 17]. To the best of our knowledge the only investigation on the decay of volatility after a crash has been performed in Ref. 18] where a power law or power law log-periodic decay of implied volatility has been observed in the S\&P500 after the 1987 financial crash. We would like to stress that implied volatilty is different from our $\gamma(t)$ because implied volatiltiy is obtained from index derivative prices by using the Black and Scholes formula instead that directly from data. Moreover the value of the exponent governing the decay of volatility is different in our study and in Ref. [18]. The analytical considerations developed above indicate that stochastic volatility models of price dynamics are able to describe the behavior of an index after a crash when they predict the volatility power-law decay in time after a crash. Therefore simple autoregressive models, such as $\operatorname{GARCH}(1,1)$ [19] models, are unable to describe the observed behavior. GARCH processes in their most compact form cannot show a scale of the stochastic process decaying as a power law after a big event. By analytical calculation and by performing numerical simulations we have shown that these models are characterized by an exponential decay of the scale of the process [20].

In order to show that empirical data are consistent with our description of aftershock periods, we empirically study the time evolution of the scale of the process. To this end, by using the ordinary least square method, we fit the absolute value of return with the functional form $f(t)=c_{1} t^{-\beta}+c_{2}$ in the 60 days after each considered market crash. We check that the relation $c_{1} t^{-\beta}>>c_{2}$ is verified in the investigated period. The best estimation of $c_{1}$ and $c_{2}$ are $6.310^{-4}$ and $2.810^{-6}$ for the $1987 \mathrm{crash}$, $5.110^{-4}$ and $4.310^{-5}$ for the 1997 crash and $4.410^{-4}$ and $1.010^{-4}$ for the $1998 \mathrm{crash}$. The time $t$ is expressed in trading day. By using the relation, $r(t)=\gamma(t) r_{s}(t)$, the $\beta$ exponent obtained is also the exponent controlling the scale $\gamma(t)$. In order to estimate the $\alpha$ exponent governing the stationary part of the return evolution we define a new variable $r_{p}(t)$ obtained dividing $r(t)$ by the moving average of its absolute value. The averaging window is set to 500 trading minutes. The quantity $r_{p}(t)$ is a proxy for the stationary return $r_{s}(t)$. We investigate the asymptotic properties for large absolute values of the stochastic process $r_{p}(t)$ by computing the Hill's estimator 21] of the process computed over the largest $1 \%$ values of $\left|r_{p}(t)\right|$. To assess the reliability of the $\alpha$ estimate obtained with this method we also compute its $95 \%$ confidence interval. The $95 \%$ confidence interval is obtained by computing $C_{95} \alpha / \sqrt{m}$ where $C_{95}$ is the value at which the normal distribution is equal to 0.95 and $m$ is the number of records located in the distribution tail. With our procedure, we obtain a value of the exponent $\alpha$ which is ranging from 3.18 to 3.67 . These values are consistent with the observations performed by different authors on the power law behavior governing large absolute returns in stocks and stock indices [16, 17].

The estimates of $\alpha$ and $\beta$ values are shown in the right part of Table I for all the investigated market crashes. The last column of the Table gives the value of the product $\alpha \beta$ that is to be compared with the values of $p$ summarized in the left part of the Table. The agreement is increasingly good for values of $p$ obtained for large values of the threshold. This is expected because only for large threshold the relevant part of the return pdf is well described by a power law behavior.

Finally, we investigate the properties of $N(t)$ computed for the random variable $r_{p}(t)$. This variable is our proxy for $r_{s}(t)$ and therefore a linear behavior of $N(t)$ is expected for each value of the threshold chosen. ¿From our definition of $r_{p}(t)$, it follows that the mean of the absolute value of $r_{p}(t)$ is equal to one. In Fig. 4, we show $N(t)$ for the market crash of 19 October 1987 when $\ell$ is ranging from 4 to 13 . For all values of the threshold, $N(t)$ is approximately linear showing that $r_{p}(t)$ provides a good proxy for $r_{s}(t)$. Moreover, starting from equation (4), one 
can show that the slope $\eta$ of $N(t)$ is proportional to the quantity $1-F_{s}(\ell)$. We determine $\eta$ with a best linear fit of $N(t)$ for each value of $\ell$. The results are shown in the inset of Fig. 4. Under the assumption of equation (5), the expected relation between $\eta$ and $\alpha$ is $\eta \sim \ell^{-\alpha}$. The inset also shows our best fit of $\eta$ with a power law relation as a solid line. The best fitting exponent is $\alpha=3.14$ when $\ell \geq 7$. This value of $\alpha$ is consistent with the value obtained with the Hill estimator (see Table I).

In conclusion our results show that time periods of the order of 60 trading days (approximately 3 months in calendar time) occurring after a major financial crash can be modeled in terms of a new stylized statistical law. Specifically, the number of index returns computed at a given time horizon occurring above a large threshold is well described by a power law function which is analogous to the Omori law of geophysics.

The presence of a power law relaxation seems to be a common behavior observed in a wide range of com- plex systems. One possibility for this common occurrence is that the Omori law is a phenomenological manifestation of underlying common microscopic mechanisms governing the dynamics of complex systems after an extreme event. An example of such mechanisms has been proposed to model the magnetization relaxation in spin glasses where it has been shown that the presence of many metastable states whose lifetime are distributed according to a broad, power law distribution implies a power law decay of the magnetization during aging [2].

\section{Acknowledgments}

Authors wish to thank INFM, ASI, MIUR and MIURFIRB research projects for financial support. F.L. thanks Vittorio Loreto for introducing him to the Omori law.
[1] F. Omori, J. Coll. Sci. Imp. Univ. Tokyo 7, 111 (1894).

[2] J.-P. Bouchaud, J. Phys. I 2, 1705 (1992).

[3] R.V. Chamberlin, Europhys. Lett. 33, 545 (1996).

[4] S. Zapperi, A. Vespignani and H.E. Stanley, Nature 388, 658 (1997).

[5] R. Metzler , E. Barkai , and J. Klafter, Phys. Rev. Lett. 82, 3563 (1999).

[6] I.V. Gopich and N. Agmon, Phys. Rev. Lett. 84, 2730 (2000).

[7] L.C. Sampaio et al., Phys. Rev. B 64, 184440 (2001).

[8] A. Johansen, Physica A 296, 539 (2001).

[9] S. Abe and N. Suzuki, preprint available at http://xxx.lanl.gov/cond-mat/0206453

[10] P.A. Samuelson, Industrial Management Rev. 4, 41 (1965).

[11] P.H. Cootner, The Random Character of Stock Market Prices (MIT Press, Cambridge MA, 1964).

[12] J. Hull and A. White, Journal of Finance 42, 281 (1987).

[13] J.Y. Campbell, A.W. Lo, and A.C. MacKinlay, The Econometrics of Financial Markets (Princeton University Press, Princeton,1997).

[14] F. Lillo, and R. N. Mantegna, Eur. Phys. J. B 20, 503 (2000).

[15] P. Embrechts, C. Klüppelberg and T. Mikosch, Modelling Extremal Events for Insurance and Finance (SpringerVerlag, Berlin Heidelberg, 1997).

[16] T. Lux, Applied Financial Economics 6, 463 (1996).

[17] P. Gopikrishnan et al, Eur. Phys. J. B 3, 139 (1998).

[18] D. Sornette, A. Johansen and J.-P. Bouchaud, J. Phys. I 6, 167 (1996).

[19] T. Bollerslev, J. Econometrics 31, 307 (1986).

[20] F. Lillo and R.N. Mantegna, preprint available at http://xxx.lanl.gov/cond-mat/0209685

[21] B. M. Hill, Annals of Statistics 3, 1163 (1975).

[22] Y. Huang, H. Saleur, C. Sammis and D. Sornette, Europhys. Lett. 41, 43 (1998).

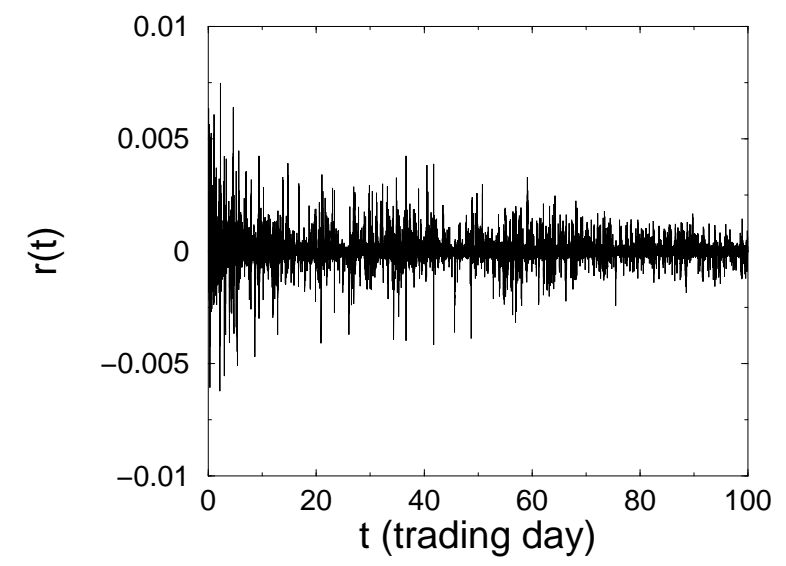

FIG. 1: One-minute change of the natural logarithm of the Standard and Poor's 500 index during the 100 trading day time period immediately after the Black Monday financial crash (20 October 1987 - 11 March 1998). A decrease of the typical scale of the stochastic process (volatility in the financial literature) is manifest making the stochastic process non-stationary. 


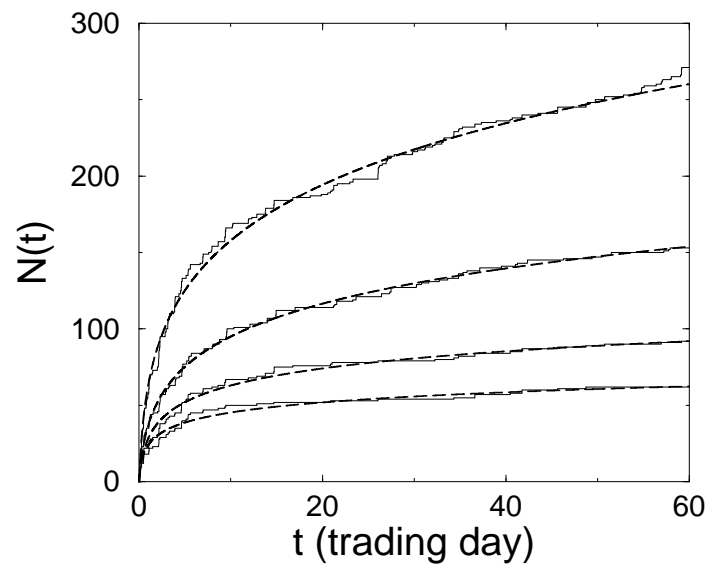

FIG. 2: Cumulative number $N(t)$ of the number of times $|r(t)|$ is exceeding a threshold $\ell$ during the 60 trading days immediately after the Black Monday financial crash. ¿From top to bottom we show the curves for values of $\ell$ equal to $4 \sigma, 5 \sigma, 6 \sigma$ and $7 \sigma$, respectively. The parameter $\sigma$ is the standard deviation of the process $r(t)$ computed over the entire investigated period. The dashed lines are best fits of equation (2).

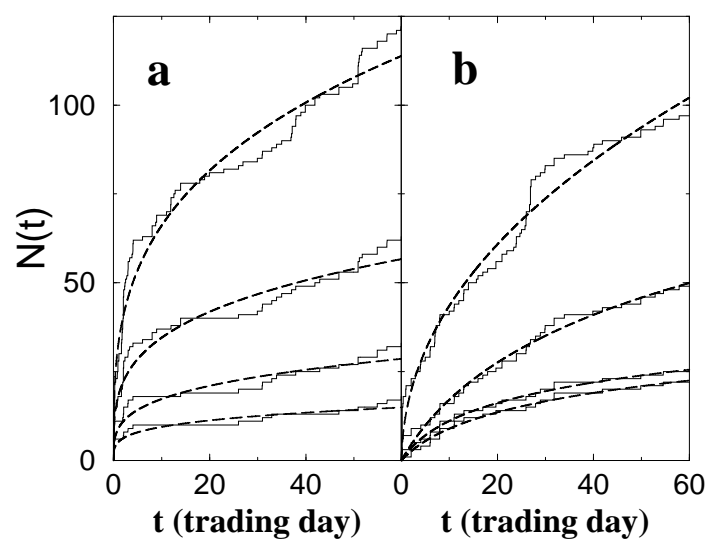

FIG. 3: Cumulative number $N(t)$ of the number of times $|r(t)|$ is exceeding the threshold $\ell$ during the 60 trading days immediately after (a) the 27 October 1997 and (b) the 31 August 1998 financial crashes. In both panels, from top to bottom we show the curves for values of $\ell$ equal to $4 \sigma, 5 \sigma, 6 \sigma$ and $7 \sigma$, respectively. The parameter $\sigma$ is the standard deviation of the process $r(t)$ computed over the entire investigated period. The dashed lines are best fits of equation (2). 


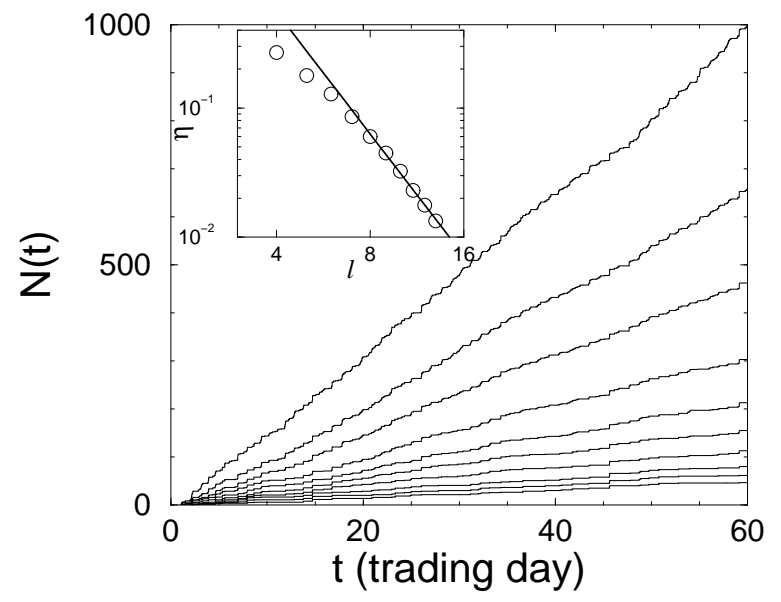

FIG. 4: Cumulative number $N(t)$ of the number of times $\left|r_{p}(t)\right|$ is exceeding a threshold $\ell$. The data refer to the $\mathrm{S} \& \mathrm{P}$ 500 index just after the 1987 crash. $N(t)$ is computed for different values of the threshold $\ell$ ranging from 4 to 13 . A linear behavior of $N(t)$ is observed for all values of $\ell$. In the inset, we show the values of the slope $\eta$ as a function of $\ell$ in a log-log plot. These values are computed by performing a best linear fitting of $N(t)$. The continuous line is the best fit of $\eta(\ell)$ with a power law behavior. The best fitting exponent is 3.14 . 